\title{
Successful treatment of refractory chronic hand eczema with calcipotriol/betamethasone ointment: A report of three cases
}

\author{
MIN YANG and JIAN-MIN CHANG \\ Department of Dermatology, Beijing Hospital, Beijing 100730, P.R. China
}

Received September 20, 2014; Accepted July 28, 2015

DOI: $10.3892 /$ etm.2015.2729

\begin{abstract}
Chronic hand eczema (CHE) is a common skin disorder with frequent relapses, and its treatment comprises a challenge due to its uncertain etiology. In particular, certain cases of CHE exhibiting severe keratinization have a very poor response to various treatments. The Daivobet ointment, a complex product comprising calcipotriol and betamethasone, has been successfully used for the treatment of patients with plaque-type psoriasis for $\sim 10$ years; however, there are few reports on the effect of the ointment on other skin disorders of abnormal keratinization, such as eczema. The present study reported 3 cases of refractory hyperkeratotic eczema of the hand that did not respond to several treatments, but responded well to topical Daivobet treatment.
\end{abstract}

\section{Introduction}

Chronic hand eczema (CHE) is a type of eczema that persists for $>3$ months or occurs at least twice a year despite adequate treatment and treatment adherence (1). CHE is a common dermatological condition with frequent relapses, occurs possibly as a result of long-term exposure to various irritants and allergens $(2,3)$. Up to $70 \%$ of cases of CHE are severe(4), and its treatment has proven to be a considerably difficult task for dermatologists. Topical use of corticosteroids, alone or in combination with keratolytic agents (such as salicylic acid and benzoic acid), retinoic acid and emollients, is the first-line treatment for CHE. Other treatment strategies, including calcineurin inhibitors and phototherapy, are used in patients with contraindications for steroids or those dissatisfied with the effects of conventional treatments. Few studies have evaluated the effectiveness of calcineurin inhibitors in hand eczema $(5,6)$. Psoralen plus ultraviolet therapy may be considered as a second-line option when topical corticosteroids are ineffective. Although its efficacy is limited, it is considered to

Correspondence to: Professor Jian-Min Chang, Department of Dermatology, Beijing Hospital, 1 Dahua Road, Beijing 100730, P.R. China

E-mail: changjm0417@126.com

Key words: hand eczema, hyperkeratotic, Daivobet be relatively safe $(3,7)$. Systemic treatments are indicated for refractory $\mathrm{CHE}$, the majority of which have not been investigated in randomized clinical trials and are therefore prescribed off-label $(2,3,8)$. At present, the only treatments approved are alitretinoin, which may be considered a second-line option as it has shown good response rates in clinical trials and observational studies (9-12). In addition, cyclosporin, azathioprine, methotrexate and mycophenolate mofetil may all be considered third-line treatments $(13,14)$. However, a poor response to various treatments is still observed in certain $\mathrm{CHE}$ cases with severe keratinization (14).

The Daivobet ointment (LEO Pharma A/S, Ballerup, Denmark), a complex product that consists of calcipotriol and betamethasone, has been proven successful in the treatment of plaque-type psoriasis during the last decade. However, the effect of Daivobet on other skin disorders of abnormal keratinization, such as eczema, remains unclear, since the literature is limited.

Subsequent to obtaining informed consent from all the patients, 3 cases of refractory hyperkeratotic eczema of the hand between February 2012 and July 2013 were successfully treated at the Department of Dermatology of Beijing Hospital (Beijing, China). In all cases, CHE was diagnosed based on the following definition: Eczema lasting for $>3$ months, or which occurs at least twice a year despite adequate treatment (1). All patients were initially topically treated with a number of corticosteroids (such as chloroflumethasone cream) alone or in combination with keratolytic agents (such as salicylic acid or benzoic acid) or retinoic acid. The therapeutic effect in each case was evaluated using photographic comparison, and each patient was evaluated by the same physician at every visit.

\section{Case report}

Case 1. A 45-year-old male patient with no history of trauma or exposure to irritants was admitted to the hospital in February 2012 presenting with itchy hyperkeratotic plaques on both hands, which had developed 2 years prior to admission. Since then, the lesion had gradually spread, thickened and hardened, and painful skin fissures had appeared during the winter. The patient had been topically treated with various corticosteroids and other agents, but little improvement was observed. Upon physical examination, the patient was found to have symmetrical hyperkeratotic erythematous plaques with a well-demarcated margin on the palms. A little excoriation 
A

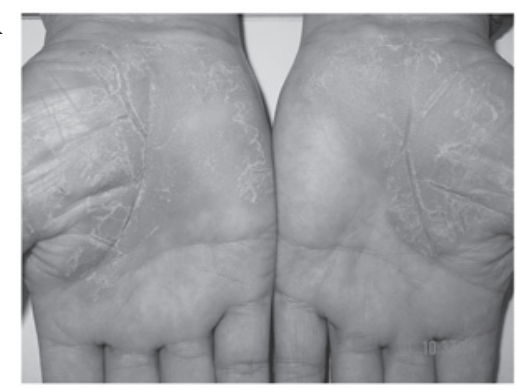

B

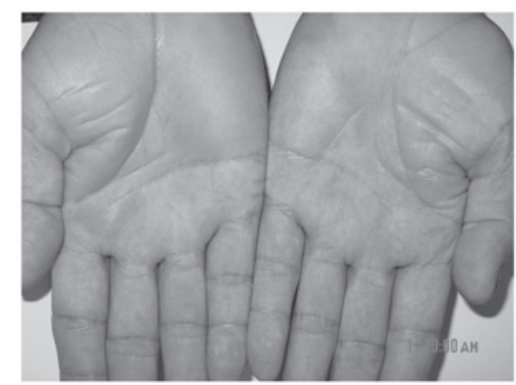

Figure 1. (A) Symmetrical hyperkeratotic erythematous plaques with a well-demarcated margin on the palms and some visible excoriation. (B) The lesions subsided completely after 16 weeks of treatment with Daivobet.

$\mathbf{A}$

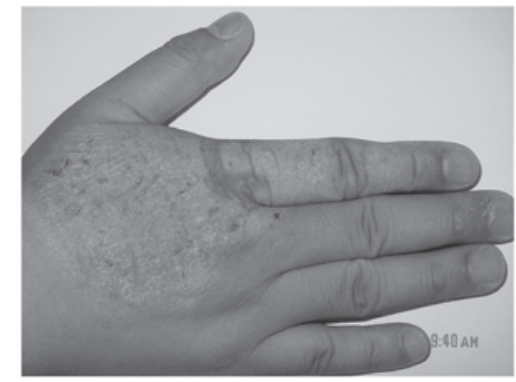

B

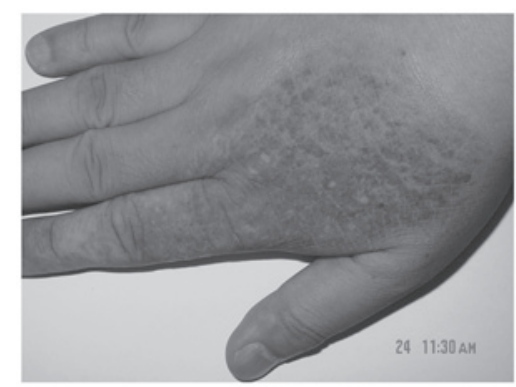

Figure 2. (A) Hyperkeratotic erythematous plaques with a well-demarcated margin on the dorsum of the right hand and some visible excoriation. (B) Non-uniform brown pigments are observed on the dorsum of the hand after 5 weeks of treatment with Daivobet.

was noted on the plaques (Fig. 1A). Treatment with $0.25 \mathrm{~g}$ Daivobet was administered twice daily. The itching subsided 2 weeks later and an almost complete regression $(90 \%)$ had been achieved by week 12. Daivobet was then applied once daily for 4 weeks. By week 16, the skin lesions had completely disappeared (Fig. 1B). No recurrence was detected at a subsequent 6-month follow-up examination following the end of treatment.

Case 2. A 44-year-old male patient was admitted in June 2012 presenting with itching hyperkeratotic plaques on the back of

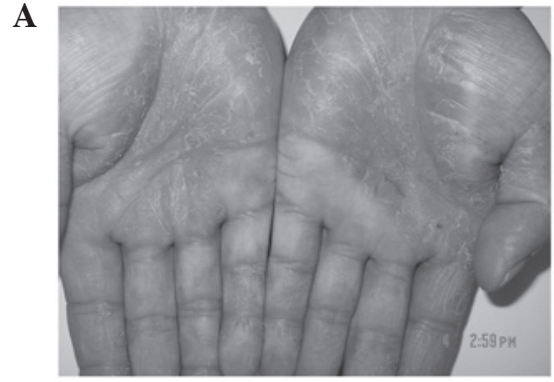

B

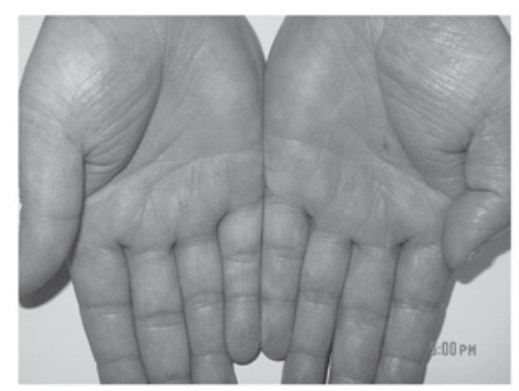

Figure 3. (A) Symmetrical hyperkeratotic erythematous plaques on both palms and flexor surface of the fingers. Scale and fissuring are observed on the plaques. (B) Almost complete resolution of lesion (90\%) after 24 weeks of treatment with Daivobet. No lesion recurrence was noted at the 2-month follow-up.

his right hand, which had developed 2 years prior to admission. The patient had previously undergone various topical therapies with no signs of improvement. Upon physical examination, hyperkeratotic erythematous plaques with a clear margin were observed on the extensor surface of the right hand of the patient, with some visible excoriation on the surface of the lesion (Fig. 2A). Daivobet was applied twice daily. The itching subsided and the lesions became darker and thinner after 1 week. The application of Daivobet was then reduced to once daily. After a further 2 weeks, the lesion had completely disappeared and only post-inflammatory pigmentation was observed (Fig. 2B). No recurrence was detected at a subsequent 6-month follow-up examination following the end of treatment.

Case 3. A 33-year-old male patient was admitted to the hospital in December 2012 presenting with itchy hyperkeratotic erythematous plaques on both palms, which had been developing for 2.5 years. Itchy, small erythematous plaques initially presented on the dorsum of the right hand. Several months later, the lesion had increased in size and extended to the left hand, and the itching had exacerbated. Various topical agents were administered, with little or no improvement. Upon physical examination, symmetrical hyperkeratotic erythematous plaques with some scaling and fissuring were observed on both palms and the flexor surface of the fingers (Fig. 3A). Daivobet was initially applied twice daily. The itching subsided after 2 weeks. Fearing possible adverse reactions, the patient decided to discontinue the use of Daivobet. There is a possibility that hypothalamo-pituitary-axis (HPA) suppression may occur if a potent corticosteroid component is absorbed to a sufficient extent. As betamethasone dipropionate is potent class 2 corticosteroid, there is a possibility that it may cause HPA axis suppression if significant systemic absorption 
occurs. After 10 weeks, the lesion and subjective symptoms had deteriorated. Subsequently, the patient began to reuse Daivobet twice daily and $80 \%$ of the lesion was resolved within 8 weeks. The application was then reduced to once daily, and $\sim 90 \%$ of the lesion was resolved within a further 4 weeks (Fig. 3B). The 2-month follow-up did not show a recurrence of the lesion.

None of the 3 patients had a history of systemic diseases, allergies to corticosteroids and vitamin D derivatives, psoriasis or other inflammatory disorders, and patch and fungal test results were negative. No other lesions, with the exception of those on the hands, were observed during the patients' visits to the hospital. All topical therapies were discontinued 2 weeks prior to the administration of Daivobet. No adverse reactions, such as contact dermatitis or HPA axis suppression, occurred during or following treatment.

\section{Discussion}

CHE is one of the most commonly diagnosed skin disorders and its treatment poses a challenge to the majority of dermatologists, due to its uncertain etiology and pathogenesis (14). Egawa (15) reported the successful treatment of 5 patients with hyperkeratotic palmoplantar eczema with a topical vitamin $\mathrm{D}_{3}$ derivative (calcipotriol or maxacalcitol). Of these cases, 4 patients were almost cured after 2-8 weeks of treatment, and in the fifth patient, the lesion improved markedly following a 7-week treatment. No adverse reactions occurred during or following the treatment, and when relapses occurred, the patients responded well to treatment (15). The successful treatment of these previous cases prompted us to attempt the application of a calcipotriol/betamethasone ointment for refractory CHE. All 3 patients in the present study were male, aged 33-45 years, with a disease course of 2-2.5 years. In 2 of the cases, the lesions were localized on the palms and in 1 case on the dorsum of the hand. The treatment duration was 5-24 weeks. In total, 2 patients were completely cured and 1 patient experienced a remarkable improvement $(90 \%$ resolution of the lesion) following treatment. The subjective symptoms (itching) in all 3 patients disappeared within 1-2 weeks.

The Daivobet ointment used for the treatment of these cases is a complex product containing the vitamin $\mathrm{D}_{3}$ derivative calcipotriol $(50 \mu \mathrm{g} / \mathrm{g})$ and the ultrapotent corticosteroid betamethasone $(0.5 \mathrm{mg} / \mathrm{g})(16)$. Vitamin $\mathrm{D}_{3}$ derivatives can bond with vitamin $\mathrm{D}$ receptors on the surface of immune cells, which contributes to the following biological effects: i) Suppression of keratinocyte proliferation and regulation of keratinocyte differentiation; ii) suppression of angiogenesis; iii) regulation of the release of cytokines, including suppression of the generation of inflammatory cytokines; and iv) regulation of inflammatory and immune responses in allergic contact dermatitis by decreasing the hapten-induced activation of the dendritic cells $(15,16)$.

The anti-inflammatory and anti-immunity effect of betamethasone is wide and non-specific; it suppresses inflammatory reactions at all stages of a disease. Furthermore, it can effectively suppress various immune responses, including the reduction of antibody generation, antigen uptake and antigen presentation through the interaction with macrophages, and inhibition of lymphocyte proliferation (17).
Thus far, Daivobet has been primarily used clinically for patients with psoriasis $(18,19)$. Two large scale randomized controlled trials confirmed that the Daivobet has numerous advantages compared with calcipotriol, tacalcitol, betamethasone and placebo, including safety, tolerance, rapid onset and effectiveness $(20,21)$. All patients with CHE who were enrolled in the present study exhibited a severe hyperkeratotic lesion and showed poor responses to corticosteroids or other agents. Psoriasis and CHE with hyperkeratinization are considered to share common histopathological features, such as marked abnormal keratinization, psoriasis-like epidermal proliferation, angiectasis of the upper dermis and chronic inflammatory cell infiltration surrounding the vessel. These common features provide histological evidence that Daivobet can be regarded as an alternative therapy for chronic refractory eczema with abnormal keratinization, when other treatments are inefficient.

In conclusion, in the present study, Daivobet was used to successfully treat 3 patients with $\mathrm{CHE}$, and thus is a safe and effective choice for the treatment of refractory CHE. However, the present study had a limited sample of cases. Therefore, large-sample randomized controlled trials supporting these results are required.

\section{References}

1. Menné T, Johansen JD, Sommerlund M and Veien NK; Danish Contact Dermatitis Group: Hand eczema guidelines based on the Danish guidelines for the diagnosis and treatment of hand eczema. Contact Dermatitis 65: 3-12, 2011.

2. Diepgen TL, Elsner P, Schliemann S, Fartasch M, Köllner A, Skudlik C, John SM and Worm M; Deutsche Dermatologische Gesellschaft: Guideline on the management of hand eczema ICD-10 Code: L20 L23. L24. L25. L30. J Dtsch Dermatol Ges 7 (Suppl 3): S1-16, 2009 (In German).

3. English J, Aldridge R, Gawkrodger DJ, Kownacki S, Statham B, White JM and Williams J: Consensus statement on the management of chronic hand eczema. Clin Exp Dermatol 34: 761-769, 2009.

4. Apfelbacher C, Molin S, Weisshaar E, Bauer A, Elsner P, Mahler V, Weiss M, Ruzicka T and Diepgen TL: Characteristics and provision of care in patients with chronic hand eczema: Updated data from the CARPE registry. Acta Derm Venereol 94: 163-167, 2014.

5. Krejci-Manwaring J, McCarty MA, Camacho F, Manuel J, Hartle J, Fleischer A Jr and Feldman SR: Topical tacrolimus $0.1 \%$ improves symptoms of hand dermatitis in patients treated with a prednisone taper. J Drugs Dermatol 7: 643-646, 2008.

6. Bauer A, Lange N, Matterne U, Meurer M, Braeutigam M and Diepgen TL: Efficacy of pimecrolimus $1 \%$ cream in the long term management of atopic hand dermatitis. A double-blind RCT. J Dtsch Dermatol Ges 10: 426-433, 2012.

7. Gritiyarangsan P, Sukhum A, Tresukosol P and Kullavanijaya P: Topical PUVA therapy for chronic hand eczema. J Dermatol 25: 299-301, 1998.

8. Lynde C, Guenther L, Diepgen TL, Sasseville D, Poulin Y, Gulliver W, Agner T, Barber K, Bissonnette R, Ho V et al: Canadian hand dermatitis management guidelines. J Cutan Med Surg 14: 267-284, 2010.

9. Schindler M, Drozdenko G, Kühl AA and Worm M: Immunomodulation in patients with chronic hand eczema treated with oral alitretinoin. Int Arch Allergy Immunol 165: 18-26, 2014.

10. Ham K, Maini P and Gooderham MJ: Real-world experience with alitretinoin in a community dermatology practice setting in patients with chronic hand dermatitis. J Cutan Med Surg 18: 332-336, 2014.

11. Gola M, Milanesi N and D'erme AM: Clinical evaluation and assessment of the therapeutic efficacy of alitretinoin in a group of patients with chronic hand eczema refractory to topical steroid therapy. G Ital Dermatol Venereol 149: 435-439, 2014. 
12. Schmith GD, Singh R, Gomeni R, Graff O, Hamedani AG, Troughton JS and Learned SM: Use of longitudinal dose-response modeling to support the efficacy and tolerability of alitretinoin in severe refractory chronic hand eczema (CHE). CPT Pharmacometrics Syst Pharmacol 4: 255-262, 2015.

13. Mrowietz U, Klein CE, Reich K, Rosenbach T, Ruzicka T, Sebastian M and Werfel T. Cyclosporine therapy in dermatology. J Dtsch Dermatol Ges7: 474-479, 2009 (In German).

14. de León FJ, Berbegal L and Silvestre JF: Management of chronic hand eczema. Actas Dermosifiliogr: May 21, 2015 (Epub ahead of print) (In Spanish).

15. Egawa K: Topical vitamin D3 derivatives in treating hyperkeratotic palmoplantar eczema: A report of five patients. J Dermatol 32: 381-386, 2005.

16. Simonsen L1, Høy G, Didriksen E, Persson J, Melchior N and Hansen J: Development of a new formulation combining calcipotriol and betamethasone dipropionate in an ointment vehicle. Drug Dev Ind Pharm 30: 1095-1102, 2004.
17. Gola M, D'Erme AM and Milanesi N: Clinical efficacy of two topical corticosteroids in the management of chronic hand eczema. G Ital Dermatol Venereol 150: 293-296, 2015.

18. VakirlisE,Kastanis AandIoannidesD:Calcipotriol/betamethasone dipropionate in the treatment of psoriasis vulgaris. Ther Clin Risk Manag 4: 141-148, 2008.

19. McCormack PL: Calcipotriol/betamethasone dipropionate: A review of its use in the treatment of psoriasis vulgaris of the trunk, limbs and scalp. Drugs 71: 709-730, 2011.

20. McCormack PL: Spotlight on calcipotriene/betamethasone dipropionate in psoriasis vulgaris of the trunk, limbs and scalp. Am J Clin Dermatol 12: 421-424, 2011.

21. Kragballe K, Austad J, Barnes L, Bibby A, de la Brassinne M, Cambazard F, Fleming C, Heikkilä H, Williams Z, Peyri Rey J, et al: Efficacy results of a 52-week, randomised, double-blind, safety study of a calcipotriol/betamethasone dipropionate two-compound product (Daivobet/Dovobet/Taclonex) in the treatment of psoriasis vulgaris. Dermatology 213: 319-326, 2006. 